\title{
Influence of Laboratory Culture Media on in vitro Growth, Adhesion, and Biofilm Formation of Pseudomonas aeruginosa and Staphylococcus aureus
}

\author{
Gayan Wijesinghe $^{a}$ Ayomi Dilhari $^{a}$ Buddhika Gayani ${ }^{b, d} \quad$ Nilwala Kottegodab-d \\ Lakshman Samaranayake $^{\mathrm{e}}$ Manjula Weerasekera ${ }^{a}$ \\ ${ }^{a}$ Department of Microbiology, Faculty of Medical Sciences, University of Sri Jayewardenepura, \\ Nugegoda, Sri Lanka; ${ }^{b}$ Department of Chemistry, Faculty of Applied Sciences, University of Sri Jayewardenepura, \\ Nugegoda, Sri Lanka; ' Institute of Nanotechnology, Center for Excellence in Nanotechnology, Nanoscience \\ and Technology Park, Homagama, Sri Lanka; ${ }^{d}$ Center for Advanced Materials Research, Faculty of Applied \\ Sciences, University of Sri Jayewardenepura, Nugegoda, Sri Lanka; ${ }^{e}$ Health Sciences Center, Kuwait University, \\ Kuwait City, Kuwait
}

\section{Significance of the Study}

- The study explored the influence of different culture media on growth, adhesion, and biofilm formation of Pseudomonas aeruginosa and Staphylococcus aureus. The data identified Brain Heart Infusion broth as the most conducive medium for the growth kinetics of these pathogens.

\section{Keywords}

Pseudomonas aeruginosa $\cdot$ Staphylococcus aureus $\cdot$ In vitro biofilm · Culture media · Monospecies · Dual species

\begin{abstract}
Objective: Pseudomonas aeruginosa and Staphylococcus aureus dual-species biofilm infections are notoriously difficult to manage. This study aimed at investigating the influence of four different culture media on the planktonic growth, adhesion, and biofilm formation of $P$. aeruginosa and S. aureus. Materials and Methods: We monitored four different culture media including Nutrient Broth, Brain Heart Infusion (BHI) broth, Luria-Bertani broth, and RPMI 1640 medium on the planktonic growth, adhesion, and biofilm formation of
\end{abstract}

P. aeruginosa (ATCC 27853) and S. aureus (ATCC 25923) using MTT assay and scanning electron microscopy (SEM). Results: The most robust growth of the mono- and dual-species cultures was noted in BHI broth. On the contrary, RPMI 1640 medium promoted maximal initial adhesion of both the mono- and dual-species, but BHI broth fostered the maximal biofilm growth. SEM images showed profuse extracellular polysaccharide production in biofilms, particularly in coculture, in BHI medium. Conclusion: Our data demonstrate that $\mathrm{BHI}$ broth, relative to the other tested media, is the most conducive for in vitro evaluation of biofilm and planktonic growth kinetics of these two pathogens, both in mono- and coculture.

(C) 2018 The Author(s)

Published by S. Karger AG, Basel

\begin{tabular}{|c|c|c|}
\hline KARGER & $\begin{array}{l}\text { () } 2018 \text { The Author(s) } \\
\text { Published by S. Karger AG, Basel }\end{array}$ & $\begin{array}{l}\text { Karger } \\
\text { Open access }\end{array}$ \\
\hline $\begin{array}{l}\text { E-Mail karger@karger.com } \\
\text { www.karger.com/mpp }\end{array}$ & $\begin{array}{l}\text { This is an Open Access article license } \\
\text { Attribution-NonCommercial- } 4.0 \text { Int } \\
\text { (http://www.karger.com/Services/Op } \\
\text { the online version of the article only. } \\
\text { mercial purposes requires written pe }\end{array}$ & $\begin{array}{l}\text { r the Creative Commons } \\
\text { nnal License (CC BY-NC) } \\
\text { essLicense), applicable to } \\
\text { and distribution for com- } \\
\text { on. }\end{array}$ \\
\hline
\end{tabular}

Dr. Manjula Weerasekera

Department of Microbiology, Faculty of Medical Sciences

University of Sri Jayewardenepura

Gangodawila, Nugegoda (Sri Lanka)

E-Mail mmweera@yahoo.com, mmweera@sjp.ac.lk 


\section{Introduction}

Chronic, recalcitrant polymicrobial infections caused by biofilms are increasingly being recognized worldwide. Such infections could be due to the colocation of extrinsically acquired putative pathogens, and members of the commensal flora whence they communicate, cooperate, and compete with each other [1]. Two important bacterial pathogens that have developed a complex network of evasion, counter-inhibition, and subjugation in their battle for space and nutrients are Pseudomonas aeruginosa and Staphylococcus aureus. For instance, in cystic fibrosis lung infections, in wound and burn infections, and catheter-associated urinary tract infections, they act either in tandem or in competition, generally leading to worse patient outcomes [2-4].

Both $P$. aeruginosa and $S$. aureus have the ability to colonize and establish biofilms, defined as a surface-attached microbial community surrounded by a self-produced exopolymer matrix [5]. Biofilm formation is a complex process which is a central pathogenic event in the infection. It involves distinct phases of attachment, accumulation, and maturation and follows a sequence of events similar to that of planktonic counterparts with lag, log, stationary, and decline phases [6]. Moreover, the biofilms of these organisms adorned with a thick coat of the extracellular polysaccharide are extremely resistant to antibiotics as this coat protects the pathogen by inhibiting its phagocytosis [7]. A number of workers have therefore evaluated monospecies and mixed species biofilm coculture models of the two pathogens using various culture media to elucidate their biofilm phenotype and physiology $[8,9]$. Nevertheless, limited data are available on the effect of culture media on growth kinetics of biofilms.

It is known that cultures of $P$. aeruginosa and $S$. aureus in such diverse media yield disparate results. Some even fail to develop biofilm complexes of bacteria found in differing niduses depending on their role within the biofilm [10]. Additionally, with the growing attention on biofilm infections and the need for finding novel therapeutic strategies to combat these infections, it is essential to standardize relevant techniques across laboratories. Yet, the culture media used for these studies differ from one laboratory to another, and yield data that are not directly comparable. In the current study, we studied three different commonly used culture media, including Nutrient Broth (NB), Brain Heart Infusion (BHI) broth, Luria-Bertani (LB) broth, and RPMI 1640 medium, which is not normally used for bacteria but for the growth of eukaryotic cells to seek the ability of biofilm formation of $P$. aeruginosa and $S$. aureus.

Influence of Culture Media on Biofilm Growth
The main objective of the current study was to determine the impact of four different culture media with varying nutrient compositions on the growth, adhesion, and biofilm formation of mono- and cocultures of $S$. aureus and $P$. aeruginosa biofilms, so as to provide foundation data for future functional studies.

\section{Materials and Methods}

\section{Test Strains and Culture Media}

P. aeruginosa (ATCC 27853) and S. aureus (ATCC 25923) type strains obtained from the American Type Culture Collection were used throughout. Stock cultures were maintained on Nutrient Agar (NA; Sigma-Aldrich, USA) slants, and sub-cultured onto freshly prepared NA plates and incubated at $35^{\circ} \mathrm{C}$ for $24 \mathrm{~h}$. For all planktonic, adhesion, and biofilm assays, NB (Sigma-Aldrich), BHI (HiMedia, India) broth, LB broth, and RPMI 1640 (Gibco, USA) medium were used as culture media.

\section{Planktonic Growth Assay}

Planktonic growth rates in different culture media were determined as described previously by Jin et al. [11] with modifications. Briefly, standard cell suspensions of $P$. aeruginosa and $S$. aureus were prepared in sterile NB, BHI broth, LB, and RPMI 1640 by adjusting the turbidity of suspensions to $0.5 \mathrm{MacF}$ arland standard. A 1:1 suspension of $P$. aeruginosa and $S$. aureus cells was also prepared. Then, $100 \mu \mathrm{L}$ of each suspension was inoculated in triplicate into a sterile, flat-bottomed, polystyrene 96-well microtiter plate. The growth rate of planktonic bacteria was determined by measuring the optical density (OD) of the suspensions in each well at $492 \mathrm{~nm}$ at 2-h intervals for $14 \mathrm{~h}$ using a microtiter plate reader (SPECTRAmaxPLUS384 Molecular Devices Inc., USA), and growth curves were generated.

\section{Bacterial Adhesion}

The cell suspensions of $P$. aeruginosa and $S$. aureus, which were equal to $0.5 \mathrm{MacFarland}$ standards, were prepared in sterile NB, BHI broth, LB, and RPMI 1640. In addition to the monospecies suspensions, 1:1 mixed suspensions were prepared by adding equal volumes of each species. Then, $100 \mu \mathrm{L} /$ well standard cell suspensions of $P$. aeruginosa, $S$. aureus, and 1:1 mixed species were added in triplicate to wells of a sterile flat-bottomed microtiter plate and incubated for $90 \mathrm{~min}$ at $37^{\circ} \mathrm{C}$ for initial adhesion studies [11]. After $90 \mathrm{~min}$ of incubation, the nonadherent cells were removed by washing plates carefully with $200 \mu \mathrm{L}$ of sterile phosphate-buffered saline (PBS) and the adherent cells were quantified using MTT assay (tetrazolium salt 3-[4, 5-dimethylthiazol-2-yl]-2, 5-diphenyltetrazolium bromide; Sigma-Aldrich) assay [12, 13], with minor modifications, as outlined below.

A working solution of $1 \mathrm{mg} / \mathrm{mL}$ MTT was initially prepared with sterile, filter-sterilized distilled water. To quantify the adherent viable cell mass, $50 \mu \mathrm{L}$ of working solution was added to each well and incubated at $37^{\circ} \mathrm{C}$ for $4 \mathrm{~h}$. After incubation, the remaining MTT solution was aspirated carefully. To solubilize the formazan end product, $100 \mu \mathrm{L}$ of dimethyl sulfoxide (Sigma-Aldrich) was added to each well, and absorbance was measured at 570 and 630 nm wavelengths. 
Fig. 1. Growth curves of planktonic P. aeruginosa, $S$. aureus, and 1:1 mixed species in the four different culture media: $\mathrm{NB}$ (a), BHI broth (b), LB (c), and RPMI 1640 (d). Data are presented as the mean $( \pm$ SD) of three independent experiments performed in triplicate.

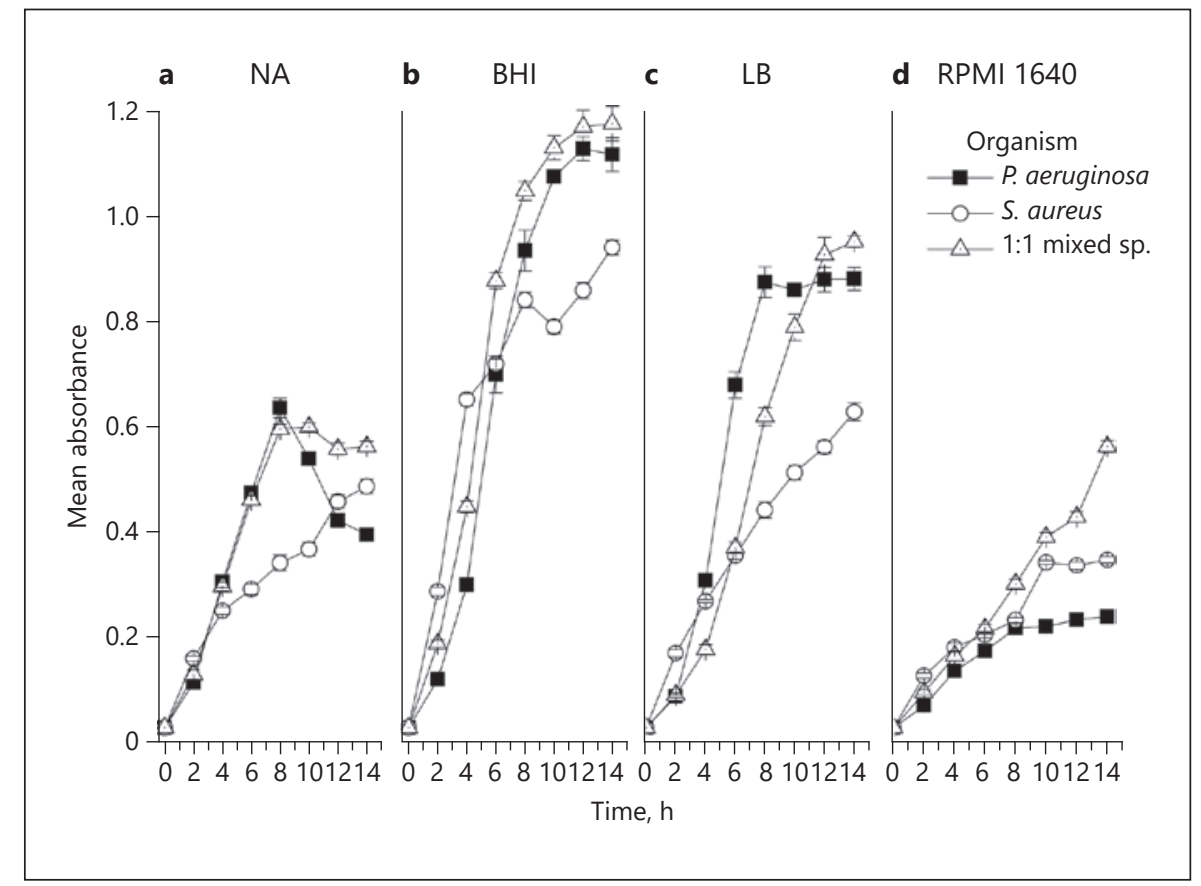

Biofilm Growth Assay

Biofilm assay was performed using the method of Jin et al. [11]. Briefly, standard cell suspensions (equal to $0.5 \mathrm{McF}$ arland turbidity standard) of $P$. aeruginosa, $S$. aureus, and 1:1 mixed species were prepared in sterile PBS and $100 \mu \mathrm{L}$ of this standard cell suspension was added in triplicate to each well of a flat-bottom sterile microtiter plate and incubated for $90 \mathrm{~min}$ at $37^{\circ} \mathrm{C}$ to achieve the initial cell adhesion. The plate was then washed twice with $200 \mu \mathrm{L} /$ well of sterile PBS to remove nonadherent cells and the wells refilled with 100 $\mu \mathrm{L} /$ well of the four different sterile culture media: NB, BHI broth, $\mathrm{LB}$, and RPMI 1640. The plate was incubated at $37^{\circ} \mathrm{C}$ up to $96 \mathrm{~h}$ and the culture media replenished daily. The biofilm biomass was quantified at 24, 48, 72, and $96 \mathrm{~h}$ using the MTT assay as described above. Growth curves were prepared to determine the effect of the four different liquid culture media on biofilm growth.

\section{Scanning Electron Microscopy}

For ultrastructural studies, the biofilms of $P$. aeruginosa, $S$. aureus, and 1:1 mixed species were developed as described above on 10 -mm diameter coverslips (which were pretreated with concentrated sulfuric acid and 95\% ethanol before the experiments), processed, and examined as follows. The biofilms were fixed with $2.5 \%$ glutaraldehyde for $2 \mathrm{~h}$ and serially dehydrated with ethanol. After overnight critical point drying in a desiccator, the biofilms were coated with gold and examined using a scanning electron microscope (SEM; Hitachi SU 6600, Tokyo, Japan).

\section{Statistics}

Each experiment was performed in triplicate. Statistical analyses were performed using SPSS16.0 for Windows. One-way and two-way ANOVA were performed to analyze the differences among multiple means. $p$ values $<0.05$ were considered statistically significant.

\section{Results}

Growth Rate of Planktonic Bacteria in Culture Media

In the NB medium, both $P$. aeruginosa and the 1:1 mixed species coculture growth reached a plateau at $8 \mathrm{~h}$, after which a decline in growth was noted. NB did not particularly support S. aureus monoculture growth, which was highly retarded compared to its counterpart (Fig. 1a). In the BHI broth medium, $P$. aeruginosa and the 1:1 profiles of mixed species coculture were similar, reaching a plateau at $12 \mathrm{~h}$, and provided the highest yield amongst all four media (Fig. 1b).

In the LB broth medium, $P$. aeruginosa growth reached a plateau at $6 \mathrm{~h}$ and $S$. aureus growth was significantly retarded, and only plateaued after $14 \mathrm{~h}$. The mixed species coculture reached a plateau at $14 \mathrm{~h}$ in LB medium with a higher yield in OD terms (Fig. 1c).

The RPMI 1640 medium was least supportive of the planktonic growth of both the mono- and cocultures (Fig. 1d). S. aureus demonstrated a continuous growth upward trajectory of growth in all culture media except RPMI 1640. In summary, on comparing the planktonic growth of the two species in different culture media, the maximum growth of all the mono- and cocultures was achieved with BHI broth (Fig. 1a-d), followed by LB medium, while NB and RPMI media were least supportive.
Wijesinghe/Dilhari/Gayani/Kottegoda/ Samaranayake/Weerasekera 


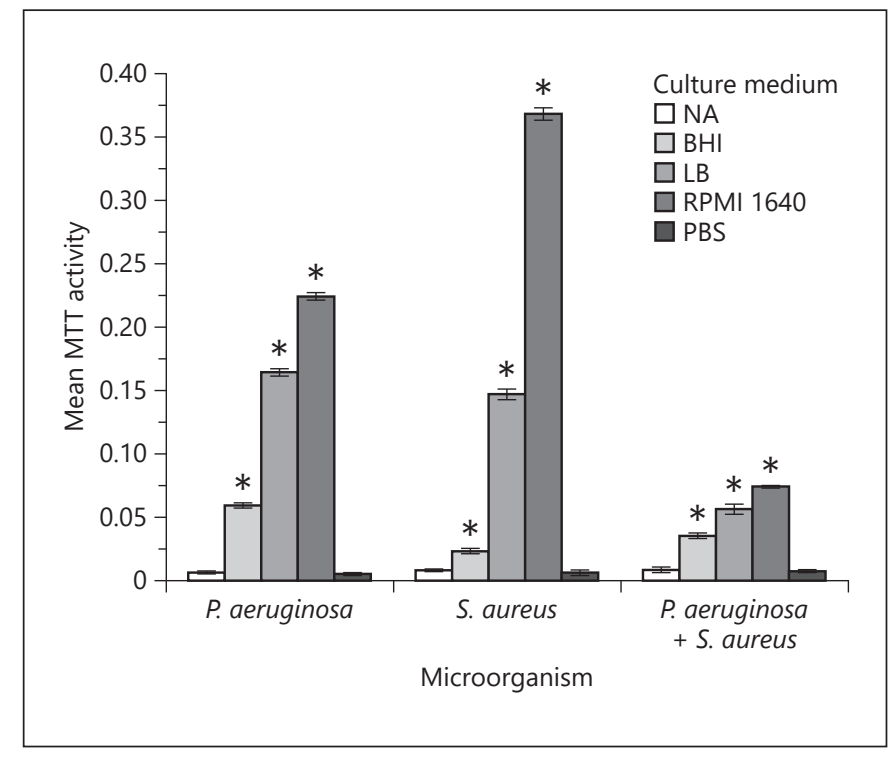

Fig. 2. Adhesion of $P$. aeruginosa, $S$. aureus, and 1:1 mixed species in NB, BHI broth, LB, and RPMI 1640 media. MTT assay was performed to quantify the adherent cell mass. Adhesion of bacterial cells in PBS served as the control. The error bars represent \pm 2 SD. For comparison of relative bacterial adhesion in different culture media, a two-way ANOVA was performed followed by a Bonferroni post hoc test. $* p<0.05$ using ANOVA, indicating a significant difference in adhesion between the four culture media for the given organism.

\section{Effect of Culture Media on Bacterial Adhesion}

MTT assays were conducted to evaluate the adhesion of $P$. aeruginosa, $S$. aureus, and 1:1 mixed species onto the plastic substrate of the multi-well plates. NB medium supported bacterial adhesion to the least extent, while the other three media promoted mono- and mixed-species adhesion, compared to the PBS control $(p<0.05)$. All mono- and mixed-species cultures elicited the highest adhesion to the substrate in RPMI 1640 medium $(p<0.05$; Fig. 2), which was far superior to either LB or BHI media.

\section{Growth of Mono- and Mixed-Species Biofilms}

Biofilm growth was quantified using the MTT assay (Fig. 3). Growth of all biofilms was uniformly high and reached a plateau at $72 \mathrm{~h}$ in all four culture media. The maximal growth of all mono- and mixed-species biofilms was noted with BHI broth, followed by RPMI 1640, LB, and NB media (Fig. 3). NB was the least supportive of biofilm growth in general. Furthermore, $P$. aeruginosa biofilm growth in NB medium was minimal compared to the other media (Fig. 3).

Influence of Culture Media on Biofilm Growth
Ultrastructure of Bacterial Biofilms in BHI Medium

As BHI broth induced maximal biofilm growth of both the mono- and cocultures of the two organisms (Fig. 3), we selected the 72-h-old biofilms in the latter medium for SEM evaluation. The individual biofilms of the P. aeruginosa and S. aureus clearly demonstrated the discrete bacterial populations attempting to form confluent microcolonies and cell islands at this stage of biofilm growth, with sparse extracellular matrix, if any. On the contrary, the coculture biofilm was characterized by relatively mature multi-layer biofilms with an extracellular polysaccharide matrix enveloping some sectors of the community (Fig. 4c, d).

Overall, the results of electron microscopy confirmed the culture results of the positive impact of the BHI medium on biofilm development of the two organisms. However, the extracellular matrix is promoted in cocultures of $P$. aeruginosa and $S$. aureus (Fig. 4b), compared to monocultures of these two organisms.

\section{Discussion}

$P$. aeruginosa and $S$. aureus are the most common agents of nosocomial infections, such as catheter-associated urinary tract infections, and wound and burn infections $[3,14]$. Additionally, they play a critical role in chronic recalcitrant infections associated with cystic fibrosis [4]. The biofilms of these two species, either singly or in combination, contribute to serious health issues due to their intrinsic resistance to antibiotics. Furthermore, conventional tests for antimicrobial sensitivity of planktonic cells do not clearly reflect the in vivo habitat of these organisms and, hence, new methods including appropriate culture media are required to yield clinically relevant data for managing these biofilm-related infections. The first step in facilitating biofilm studies is to standardize culture techniques across clinical and research laboratories.

According to our findings, BHI medium facilitates both S. aureus and P. aeruginosa planktonic as well as biofilm growth compared to the other three tested media. BHI medium in comparison to the others contains higher levels of peptones, proteins, and salts [15], which appear to facilitate planktonic growth and the biofilm yield of the two species. Protease peptone and infusions (calf brain and beef heart) provide the necessary nitrogen compounds, carbon, essential growth factors, amino acids, and vitamins. Dextrose serves as a source of energy, and sodium chloride maintains the osmotic equilibrium 
Fig. 3. Relative biofilm formation of $P$. aeruginosa, $S$. aureus, and 1:1 mixed species in the four different culture media over a 96-h period. Biofilm biomass was quantified using the MTT assay. Data are mean \pm $\mathrm{SD}$ of three separate independent experiments performed in triplicate. The error bars represent \pm 2 SD.

Fig. 4. a Scanning electron micrograph of a 72-h-old 1:1 mixed species biofilm in BHI medium (the scale indicates $20.0 \mu \mathrm{m}$ ). b Scanning electron micrograph of a 72-hold 1:1 mixed species biofilm of $P$. aeruginosa and $S$. aureus in BHI medium (the scale indicates $5.0 \mu \mathrm{m}$ ). The SEM image shows significant production of extracellular polymeric matrix. c Scanning electron micrograph of a 72-h-old $P$. aeruginosa monospecies biofilm in BHI medium (the scale indicates $20.0 \mu \mathrm{m})$. d Scanning electron micrograph of a 72 -h-old $S$. aureus monospecies biofilm in BHI medium (the scale indicates $10.0 \mu \mathrm{m})$.
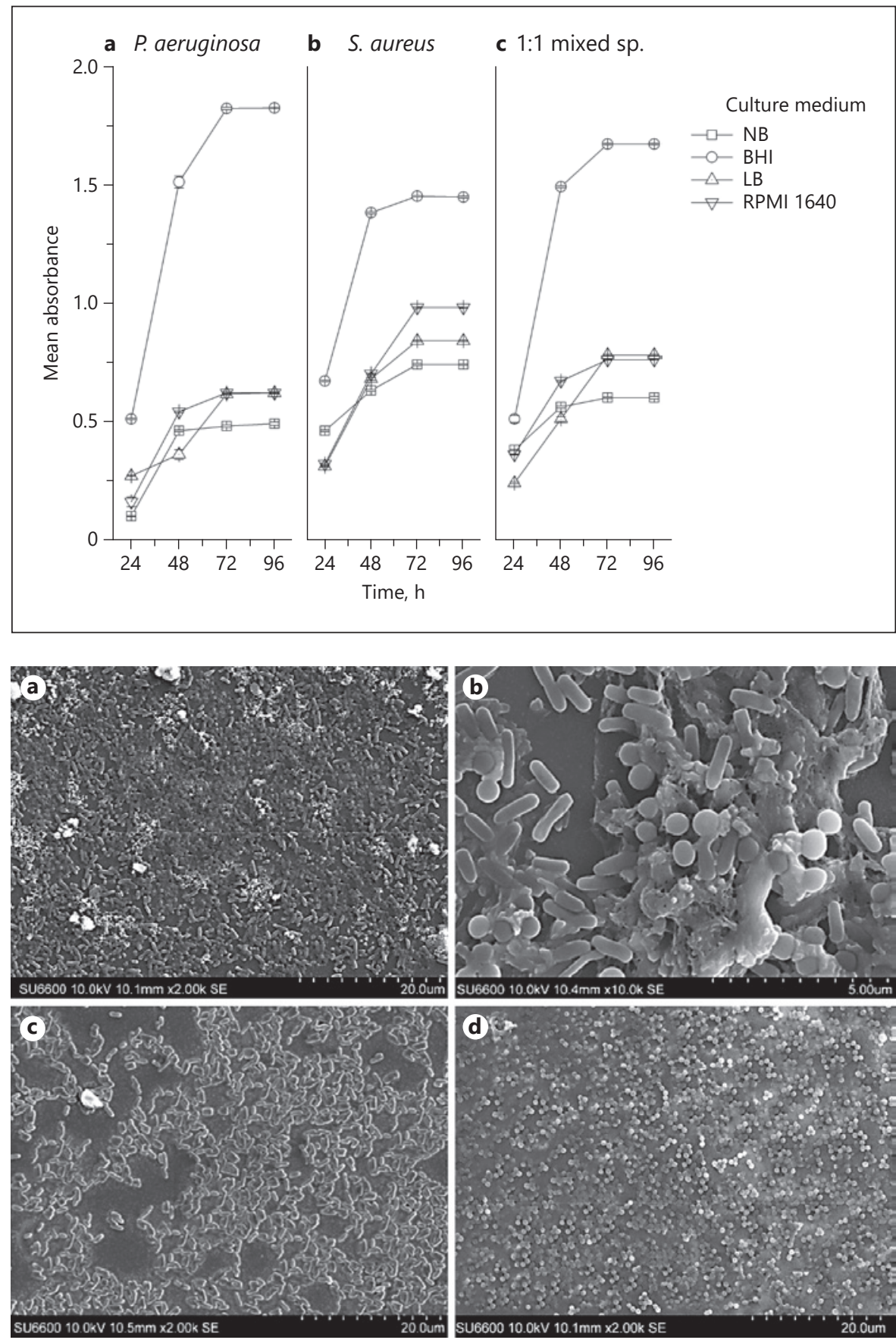

of the medium. Others have previously noted that the nutrient composition of the culture medium has a major impact on biofilm growth and development [16]. Our data to some extent confirms the findings of a recent study by Chen et al. [17], who recommended BHI as a good culture medium for the growth of $S$. aureus biofilms.
In the NB medium, only the beef extract and peptone provide the necessary nitrogen, carbon and vitamins, while sodium chloride maintains osmotic equilibrium of the medium. In LB medium, peptone provides basic nutrients and growth factors to the bacteria. Yeast extract supplies vitamins, amino acids, and trace elements. Sodium chloride maintains the proper isotonic environ- 
ment of the broth. Based on their composition, the concentration of nutrients is highest in BHI, followed by LB and NB.

The adherent capacities of different species to the various substrata are disparate, and are facilitated by several factors, including the culture medium [18]. For instance, Hood and Zottola [19] studied four different bacterial species, including Salmonella typhimurium, Listeria monocytogenes, Escherichia coli O157:H7, Pseudomonas fragi, and P. fluorescens, and concluded that the medium which produced the highest level of adherent cells was different for each organism. It was also shown that the lack of nutrients could enhance the adhesion of some microorganisms, while others exhibited high adhesion rates even under basic growth conditions [20]. We noted that the adherence of both species, either in mono- or cocultures, compared with the other three media, was optimal in RPMI 1640. The latter medium mimics the composition of human body fluids and contains high concentrations of amino acids, including L-glutamine, L-arginine, $\mathrm{L}$-asparagine, as well as vitamins and inorganic salts [21]. It is tempting to speculate, therefore, that the rich RPMI 1640 medium replete with amino acids may have induced the two species to develop extracellular surface components favorable for adhesion. However, RPMI 1640 performed worst in terms of support for growth. Though RPMI 1640 is a rich nutrient medium, its amino acid composition is higher than that of carbohydrates. During the period of rapid growth, carbohydrates initially serve as the primary source of carbon and energy but are quickly spent, and other nutrients such as peptides, amino acids, nucleic acids, nucleotides, and fatty acids are utilized to sustain growth $[22,23]$.

As the carbohydrate supply is exhausted, amino acid catabolism predominates and, as cells enter the stationary phase, ammonia released into the medium causes it to become basic [24]. To survive under these conditions, cells must adapt to a high energy expenditure that is imposed on them by the need to actively acquire protons from the basic medium environment in order to maintain $\mathrm{pH}$ homeostasis in the cytoplasm [25]. Eventually, the readily available nutrients are exhausted and the only nutrients available are those derived from the dead bacteria [26]. In order to scavenge nutrients from the bacterial debris, proteins, nucleic acids, and lipids must be broken down into their constituent parts, which imposes another large energy cost on the cell. Thus, such stress responses are associated with the appearance of macromolecular agents in the medium and this could affect the viable cell mass. Therefore, this could be a major cause of the reduc-

Influence of Culture Media on Biofilm Growth tion in viable cell mass in biofilm formation in RPMI 1640 medium.

The coculture showed suppressed adhesion (Fig. 2) compared to monocultures. In a coculture biofilm the adhesion is mediated by many organism-related factors, such as quorum sensing, inhibitory factors, and environmental factors. S. aureus and $P$. aeruginosa are likely to produce biosurfactants that could inhibit their attachment or limit cell adhesion to polystyrene. These biosurfactants could have enzymatic action resulting in the degradation of biofilm polymers leading to biofilm detachment. It is known that biofilm detachment is controlled by quorum sensing [27], but the quorum sensing-controlled molecular detachment factors of $S$. aureus and $P$. aeruginosa remain undefined [28]. This could be a main reason for the suppression of adhesion in coculture compared to monocultures.

In the stage of biofilm formation and maturation, $\mathrm{BHI}$ enhanced the growth of biofilms possibly due to the high nutrient composition of the broth. The growth of the biofilms of $S$. aureus, $P$. aeruginosa, and 1:1 mixed species was more than twice that of the other three tested media. Therefore, the growth of coculture probably represents the growth rate of $P$. aeruginosa (Fig. 1). Thus, it is possible that $P$. aeruginosa facilitates microcolony formation of $S$. aureus and outcompetes $S$. aureus in coculture biofilms. This was further substantiated by high-resolution SEM. Yang et al. [29] studied the role of eDNA in P. aeruginosa and $S$. aureus mixed-species microcolony formation in coculture biofilms, emphasizing the importance of eDNA as a common biofilm EPS constituent. They showed that eDNA behaves as an essential EPS element shared by different species in coculture biofilms, which facilitates interspecies interactions through the formation of mixed-species compact microcolony structures during biofilm development. Furthermore, P. aeruginosa is able to protect $S$. aureus from phagocytosis in coculture biofilms [29].

$P$. aeruginosa and $S$. aureus are ubiquitous pathogens that cause many chronic, diabetic, and combat-wound infections, often found together in coinfections; this has led to the concept of polymicrobial biofilm-associated infections. Dean et al. [30] analyzed mixed biofilm of S. aureus and $P$. aeruginosa by laser ablation electrospray ionization mass spectrometry, and found that $S$. aureus and $P$. aeruginosa within the mixed biofilm showed significant colocalization of the cells, rather than self-segregation. Thus, the growth rate of coculture biofilms could be in between the growth rate of monospecies biofilms (that is, greater than $P$. aeruginosa and less than $S$. aureus), according to the findings of the current study. 
Finally, we noted in our ultrastructural studies that the dual-species biofilm produced profuse extracellular polysaccharide compared with the monospecies biofilms. This phenomenon has been described in wound infections coinfected with $S$. aureus and $P$. aeruginosa [2] and has important clinical implications, including recalcitrance to common antibiotics.

\section{Conclusion}

The current data demonstrate that, of the commonly used media, BHI broth is the most conducive growth medium for studying in vitro biofilm and planktonic growth kinetics of $P$. aeruginosa and $S$. aureus, particularly in coculture.

\section{Acknowledgements}

The authors wish to thank the staff members of the Department of Microbiology, Faculty of Medical Sciences, University of Sri Jayewardenepura, Sri Lanka, and Sri Lanka Institute of Nanotechnology, Center for Excellence in Nanotechnology, Nanoscience and Technology Park, Sri Lanka, for providing laboratory facilities.

\section{Disclosure Statement}

The authors have no competing interests to declare.

\section{References}

1 Hotterbeekx A, Kumar-Singh S, Goossens H, Malhotra-Kumar S. In vivo and in vitro interactions between Pseudomonas aeruginosa and Staphylococcus spp. Front Cell Infect Microbiol. 2017 Apr;7:106.

2 DeLeon S, Clinton A, Fowler H, Everett J, Horswill AR, Rumbaugh KP. Synergistic interactions of Pseudomonas aeruginosa and Staphylococcus aureus in an in vitro wound model. Infect Immun. 2014 Nov;82(11): 4718-28.

3 Fazli M, Bjarnsholt T, Kirketerp-Møller K, Jørgensen B, Andersen AS, Krogfelt KA, et al. Nonrandom distribution of Pseudomonas aeruginosa and Staphylococcus aureus in chronic wounds. J Clin Microbiol. 2009 Dec; 47(12):4084-9.

4 Saiman L, Marshall BC, Mayer-Hamblett N, Burns JL, Quittner AL, Cibene DA, et al.; Macrolide Study Group. Azithromycin in patients with cystic fibrosis chronically infected with Pseudomonas aeruginosa: a randomized controlled trial. JAMA. 2003 Oct;290(13): 1749-56.

5 Flemming HC, Wingender J. The biofilm matrix. Nat Rev Microbiol. 2010 Sep;8(9):62333.

6 Seneviratne CJ, Yip JW, Chang JW, Zhang CF, Samaranayake LP. Effect of culture media and nutrients on biofilm growth kinetics of laboratory and clinical strains of Enterococcus faecalis. Arch Oral Biol. 2013 Oct;58(10): 1327-34.

7 Davies D. Understanding biofilm resistance to antibacterial agents. Nat Rev Drug Discov. $2003 \mathrm{Feb} ; 2(2): 114-22$.

8 Yang L, Liu Y, Wu H, Hóiby N, Molin S, Song ZJ. Current understanding of multi-species biofilms. Int J Oral Sci. 2011 Apr;3(2):74-81.
9 Young KD. The selective value of bacterial shape. Microbiol Mol Biol Rev. 2006 Sep; 70(3):660-703.

10 Weerasekera MM, Wijesinghe GK, Jayarathna TA, Gunasekara CP, Fernando N, Kottegoda $\mathrm{N}$, et al. Culture media profoundly affect Candida albicans and Candida tropicalis growth, adhesion and biofilm development. Mem Inst Oswaldo Cruz. 2016 Nov;111(11): 697-702.

11 Jin Y, Samaranayake LP, Samaranayake Y, Yip HK. Biofilm formation of Candida albicans is variably affected by saliva and dietary sugars. Arch Oral Biol. 2004 Oct;49(10):78998.

12 Traba C, Liang JF. Susceptibility of Staphylococcus aureus biofilms to reactive discharge gases. Biofouling. 2011 Aug;27(7):763-72.

13 Weerasekera MM, Jayarathna TA, Wijesinghe GK, Gunasekara CP, Fernando N, Kottegoda N, et al. The Effect of Nutritive and NonNutritive Sweeteners on the Growth, Adhesion, and Biofilm Formation of Candida albicans and Candida tropicalis. Med Princ Pract. 2017;26(6):554-60.

14 Maki DG, Tambyah PA. Engineering out the risk for infection with urinary catheters. Emerg Infect Dis. 2001 Mar-Apr;7(2):342-7.

15 Petinate SD, Martins RM, Coelho RR, Meirelles $\mathrm{MN}$, Branquinha $\mathrm{MH}$, Vermelho $\mathrm{AB}$. Influence of growth medium in proteinase and pigment production by Streptomyces cyaneus. Mem Inst Oswaldo Cruz. 1999 MarApr;94(2):173-7.
16 Stepanović S, Vuković D, Hola V, Di Bonaventura G, Djukić S, Cirković I, et al. Quantification of biofilm in microtiter plates: overview of testing conditions and practical recommendations for assessment of biofilm production by staphylococci. APMIS. 2007 Aug;115(8):891-9.

17 Chen P, Abercrombie JJ, Jeffrey NR, Leung KP. An improved medium for growing Staphylococcus aureus biofilm. J Microbiol Methods. 2012 Aug;90(2):115-8.

18 Samaranayake LP, Ellepola AN. Studying Candida albicans adhesion. In: An Y, Friedman RJ, editors. Handbook of bacterial adhesion: principles, methods and applications. New York: Humana Press; 2000. p. 527-40. https://doi.org/10.1385/1-59259-224-4:527.

19 Hood SK, Zottola EA. Adherence to stainless steel by foodborne microorganisms during growth in model food systems. Int J Food Microbiol. 1997 Jul;37(2-3):145-53.

20 van Loosdrecht MC, Lyklema J, Norde W, Schraa G, Zehnder AJ. Electrophoretic mobility and hydrophobicity as a measured to predict the initial steps of bacterial adhesion. Appl Environ Microbiol. 1987 Aug;53(8): 1898-901.

21 Kucharíková S, Tournu H, Lagrou K, Van Dijck P, Bujdáková H. Detailed comparison of Candida albicans and Candida glabrata biofilms under different conditions and their susceptibility to caspofungin and anidulafungin. J Med Microbiol. 2011 Sep;60(Pt 9): 1261-9.

22 Clark DP, Cronan JE. Two-carbon compounds and fatty acids as carbon sources. Escherichia coli and Salmonella: cellular and molecular biology. 2nd ed. Washington (DC) ASM Press; 1996. pp. 343-57. 
23 Finkel SE, Kolter R. DNA as a nutrient: novel role for bacterial competence gene homologs. J Bacteriol. 2001 Nov;183(21):6288-93.

24 Bodini S, Nunziangeli L, Santori F. Influence of amino acids on low-density Escherichia coli responses to nutrient downshifts. J Bacteriol. 2007 Apr;189(8):3099-105.

25 Slonczewski JL, Rosen BP, Alger JR, Macnab RM. pH homeostasis in Escherichia coli: measurement by $31 \mathrm{P}$ nuclear magnetic resonance of methylphosphonate and phosphate. Proc Natl Acad Sci USA. 1981 Oct;78(10):6271-5.
26 Zinser ER, Kolter R. Mutations enhancing amino acid catabolism confer a growth advantage in stationary phase. J Bacteriol. 1999 Sep;181(18):5800-7.

27 Vuong C, Saenz HL, Götz F, Otto M. Impact of the agr quorum-sensing system on adherence to polystyrene in Staphylococcus aureus. J Infect Dis. 2000 Dec;182(6):1688-93.

28 Periasamy S, Joo HS, Duong AC, Bach TH, Tan VY, Chatterjee SS, et al. How Staphylococcus aureus biofilms develop their characteristic structure. Proc Natl Acad Sci USA. 2012 Jan;109(4):1281-6.
29 Yang L, Liu Y, Markussen T, Høiby N, TolkerNielsen T, Molin S. Pattern differentiation in co-culture biofilms formed by Staphylococcus aureus and Pseudomonas aeruginosa. FEMS Immunol Med Microbiol. 2011 Aug; 62(3):339-47.

30 Dean SN, Walsh C, Goodman H, van Hoek ML. Analysis of mixed biofilm (Staphylococcus aureus and Pseudomonas aeruginosa) by laser ablation electrospray ionization mass spectrometry. Biofouling. 2015;31(2):151-61. 